\title{
Etude de quelques critères indicateurs de l'état hydrique d'une culture de tomate en région semi- aride
}

\author{
Nader KATERJI, Bernard ITIER, I. FERREIRA (*) \\ avec la collaboration technique de D. Flura, B. Durand, I. Rosa \\ I.N.R.A., Station de Bioclimatologie, Centre de Recherches de Paris-Grignon, F 78850 Thiverval Grignon \\ (*) Instituto Superior de Agronomia. Tapada da Ajuda, Lisbonne (Portugal)
}

RÉSUMÉ

\begin{abstract}
Une expérience réalisée à Coruche (Portugal) sur une culture de tomate a permis de tester plusieurs critères de contrainte hydrique relatifs à la plante et à son microclimat.

Le potentiel hydrique de base et la conductance stomatique sont des critères très sensibles à un arrêt de l'irrigation. Les critères microclimatiques (température et flux de surface) peuvent être intéressants, si certaines précautions relatives à la dimension de la parcelle étudiée sont prises.

Le critère le plus sensible et le plus simple à déterminer est cependant le potentiel de base. On suggère par conséquent, de l'utiliser pour diagnostiquer les périodes de contrainte hydrique chez les plantes cultivées dans des conditions expérimentales voisines de celles étudiées ici.
\end{abstract}

Mots clés additionnels : Potentiel hydrique foliaire, température radiative, résistance stomatique, rapport du fux de chaleur sensible au rayonnement net, tomate, irrigation.

A study of several indicators of the water status of a tomato crop in a semi-arid region.

\begin{abstract}
An experiment conducted on a tomato crop at Coruche (Portugal) enabled the authors to test several water stress indicators for the plant and its microclimate. Predawn leaf water potential and stomatal conductance were very sensitive indicators of water stress. Microclimatic criteria (crop radiative temperature and parameters referring to fluxes above the canopy) could be useful provided certain precautions on the dimensions of the plot are taken. The most sensitive and easily determined of the indicators was, however, predawn leaf water potential. We therefore suggest its use as an appropriate diagnostic measure for water stress in cultivated plants grown under experimental conditions close to those studied here.
\end{abstract}

Additional key words : Leaf water potential, radiative temperature, stomatal conductance, ratio of sensible heat to net radiation, tomato crop, irrigation.

\section{INTRODUCTION}

La production des plantes cultivées décroît rapidement si leur état hydrique s'écarte d'une valeur seuil (cf. la revue bibliographique de LudLow, 1976). Autrement dit, le contrôle de l'état hydrique des végétaux d'une culture est un outil de décision pour le déclenchement de l'irrigation et par conséquent l'un des facteurs essentiels pour la réussite de celle-ci.

Pour approcher l'état hydrique du couvert, les auteurs se réfèrent à 2 types de grandeurs :

Les grandeurs, que l'on désigne ici par A, sont celles qui caractérisent directement ou indirectement l'état hydrique du couvert :
- soit en exprimant l'état hydrique de la plante. C'est le cas de la teneur en eau des feuilles (NAMKEN, 1965), ou le potentiel hydrique foliaire (MEYER \& GREEN, 1980).

- soit en traduisant la modification des différents phénomènes provoquée par le changement de cet état. Parmi ces phénomènes, on peut citer la conductance stomatique (CLARK \& HILER, 1973), les températures foliaires (cf. la revue de JACKSON, 1982), les variations de diamètre des organes végétaux (Kozlowski, 1968; NAMKEN et al., 1971) et les symptômes visuels (enroulement des feuilles) observés particulièrement chez les céréales (O’TOOLE \& MOYA, 1978).

- soit enfin, en permettant d'apprécier, sur des laps 
de temps plus ou moins longs (jour, décade) dans quelle mesure les besoins en eau de la culture ont été satisfaits. C'est le cas du rapport des évapotranspirations réelle et potentielle ETR/ETP (BOUCHET \& ROBELIN, 1969), de la fraction d'énergie disponible $\mathrm{Rn}$ utilisée dans l'ívapotranspiration réelle ETR/Rn (RITCHIE \& JORDAN, 1972) ou dans le flux de chaleur convectif H/Rn (ITIER \& RiOU, 1985).

Les grandeurs que l'on désigne ici par B sont celles qui caractérisent le milieu et conditionnent l'équilibre hydrique de la plante. Les plus importantes de ces grandeurs sont la demande climatique ETP, le stock d'eau disponible dans le sol, le potentiel hydrique dans la zone racinaire.

De nombreuses études (Bordvosky et al., 1974; STEGMAN et al., 1976) ont montré que l'utilisation des grandeurs $\mathrm{du}$ type $\mathrm{A}$ pour déclencher l'irrigation augmente l'efficience de l'eau par rapport à un déclenchement fondé sur des mesures au niveau du sol (grandeurs du type B). Sur le plan pratique, cela se traduit par une diminution du coût de l'irrigation (économie d'eau, de main-d'œuvre, d'énergie et d'engrais) et des risques de pollution des nappes phréatiques.

Toutefois les auteurs ne semblent pas être d'accord sur le critère de type A le mieux adapté pour déclencher l'irrigation (GARNiER, 1985). Pour O'ToOLE et al. (1984), ce critère est le CWSI (critère relatif à la température foliaire qui sera explicité par la suite); pour d'autres, c'est le potentiel hydrique foliaire (CLARK \& Hiler, 1973) ou la variation de diamètre des organes végétaux (GARNIER, 1985) qui semblent être les critères les plus performants. Chez la tomate, KamGAR et al. (1980) concluent qu'aucun des indicateurs testés (potentiel hydrique foliaire, conductance stomatique, température foliaire) n'est suffisamment sensible pour déclencher l'irrigation.

De plus, la plupart des grandeurs du type A restent d'accès difficile, soit parce qu'elles exigent des techniques lourdes, soit parce qu'elles requièrent la réalisation d'un grand nombre de mesures (6 à 8 mesures horaires sont nécessaires pour déterminer des valeurs journalières) lesquelles de surcroît ne sont pas automatisables.

Sur la base d'une expérimentation réalisée sur une culture de tomate en région semi-aride (Portugal), nous proposons dans la présente étude :

- de retenir 8 critères simples (particulièrement adaptés aux conditions du champ) dérivés des grandeırs du type A citées précédemment. La simplification que nous proposons et qui permet de pallier les difficultés décrites plus haut, consiste à remplacer les enregistrements continus par une mesure unique réalisée à une période de la journée où les indicateurs traduisent le mieux l'état hydrique de la plante ;

- de tester la sensibilité des critères adoptés, en comparant leur évolution sur 2 parcelles, l'une irriguée et l'autre laissée en dessèchement;

- de comparer ces critères selon leur aptitude à détecter l'état hydrique de l'espèce étudiée.

\section{MATÉRIEL ET MÉTHODES}

Les résultats que nous présentons ici ont été obtenus au cours d'une campagne de mesures réalisée à Coruche (70 km à l'est de Lisbonne) sur une culture de tomate pendant 12 jours (4-15 juillet 1985).

\section{A. L'expérimentation sur tomate}

La culture de tomate étudiée (variété H30) se trouvait en phase de floraison au début de l'expérimentation. Pour cette culture, les mesures de l'indice foliaire et de la taille des plantes sont présentées dans FERREIRA (1987). Les résultats obtenus indiquent que les 2 paramètres en question ont atteint leurs valeurs maximales (taille des plantes $=50 \mathrm{~cm}, \mathrm{LAI}=2,4)$ pendant la $1^{\text {re }}$ décade $\mathrm{du}$ mois de juillet, c'est-à-dire au début de cette expérimentation.

Le climat de la région est de type méditerranéen caractérisé par une période estivale sèche et chaude. Les données climatiques ont été recueillies par une station météorologique située à côté du dispositif expérimental. Le tableau 1 les présente pour la période de mesure. On note que cette période était sèche, chaude et caractérisée par une évaporation potentielle élevée.

La description des sols du périmètre de Coruche se trouve dans Ferreira (1987). L'analyse granulométrique indique des taux de 90 p. 100,7 p. 100,3 p. 100 respectivement pour les sable, limon et argile. Les caractères hydriques des sols ont été déterminés à partir de l'analyse des courbes potentiel-humidité au laboratoire. Ces résultats indiquent que les humidités volumiques relatives à la capacité au champ et au point de flétrissement sont respectivement de $21 \mathrm{p} .100$ et 9 p. 100. Des observations réalisées au cours de l'expérience ont montré une présence exclusive des racines dans les 40 premiers centimètres du sol. Les réserves

TABLEAU 1

Données climatologiques observées au cours de la période du I au 15 juillet 1985 à Coruche (Portugal). Climatological data observed during the period July $1-151985$ at Coruche (Portugal).

\begin{tabular}{|c|c|c|c|c|c|c|c|c|c|c|c|c|c|c|c|}
\hline Date & 1 & 2 & 3 & 4 & 5 & 6 & 7 & 8 & 9 & 10 & 11 & 12 & 13 & 14 & 15 \\
\hline T. $\min \left({ }^{\circ} \mathrm{C}\right)$ & 11,5 & 15,3 & 14,8 & 15,3 & 14,3 & 16,7 & 16.0 & 18,1 & 14.7 & 13.7 & 17,7 & 14,2 & 16,7 & 13,4 & 14,9 \\
\hline T. $\max \left({ }^{\circ} \mathrm{C}\right)$ & 29,0 & 22,8 & 24,4 & 28,7 & 34,8 & 36.7 & 34,4 & 27,3 & 27,8 & 29,7 & 29,6 & 29,7 & 39,9 & 27,6 & 37,8 \\
\hline Pluie $\left(\mathrm{mm} \cdot \mathrm{j}^{\prime}\right)$ & 0 & 0,4 & 6,7 & 0 & 0 & 0 & 0 & 0 & 0 & 0 & 0 & 0 & 0 & 0 & 0 \\
\hline Evaporation classe $A\left(\mathrm{~mm} \cdot \mathrm{j}^{\mathrm{l}}\right)$ & 7,9 & 7,1 & - & 4,7 & 6,4 & 7,1 & 6,9 & 7,4 & 6,1 & 6,1 & 5,2 & 5,1 & 4,9 & 5,6 & 7,3 \\
\hline Vitesse du vent à $15 \mathrm{~h}\left(\mathrm{~m} \cdot \mathrm{s}^{1}\right)$ & - & - & - & 1,81 & 2,32 & 1,19 & 2,32 & 2,97 & 3,03 & 2,24 & 1,13 & 1,50 & 1,80 & 1,97 & 3,25 \\
\hline
\end{tabular}


hydriques utiles dans la zone d'activité racinaire (48 $\mathrm{mm}$ ) sont donc faibles compte tenu des conditions climatiques observées dans la région.

\section{B. Méthodes et techniques employées pour déterminer les critères étudiés}

\section{Mesure de la conductance stomatique}

Les mesures de la conductance stomatique, réalisées toutes les heures à l'aide d'un poromètre à diffusion de vapeur d'eau (Automatic porometer MKII), ont porté sur des feuilles bien dégagées de la strate supérieure de la végétation. La conductance moyenne est évaluée par la moyenne arithmétique de 10 conductances ponctuelles.

\section{Mesure du potentiel hydrique foliaire $\psi_{F}$}

Des mesures horaires du potentiel foliaire sont également effectuées sur des feuilles placées dans la même situation (bien éclairées, strate supérieure). Dix feuilles sont prélevées à chaque heure et leur potentiel est immédiatement mesuré dans une chambre à pression (SCHOLANDER et al., 1965) placée à proximité de la parcelle expérimentale. La durée totale d'une mesure est de l'ordre de 2 min.

\section{Mesure de la teneur en eau des feuilles}

Les valeurs horaires de la teneur en eau des feuilles (masse d'eau rapportée à la masse de matière sèche) étaient déterminées au moyen de 10 feuilles prélevées au hasard au champ et pesées avant et après passage à l'étuve pendant $24 \mathrm{~h}$.

\section{Détermination de la fraction de l'énergie Rn utilisée dans le flux de chaleur convectif $H$}

Deux méthodes ont été utilisées pour déterminer le rapport $\mathrm{H} / \mathrm{Rn}$ :

- La première, plus élaborée, consiste à mesurer le rayonnement net par un bilanmètre et à calculer le flux $\mathrm{H}$ par la méthode aérodynamique combinée (ITIER, 1981). Rappelons que ce calcul nécessite la mesure de la vitesse du vent et du gradient thermique entre 2 niveaux fixes au-dessus de la végétation.

- La deuxième, plus simple, permet d'apprécier le rapport $\mathrm{H} / \mathrm{Rn}$ en s'appuyant simplement sur une mesure de gradient thermique $\Delta \mathrm{T}$ dans l'air. Cette méthode a été proposée par ITIER \& RIOU (1985) compte-tenu qu'en milieu de journée, en zone aride ou semi-aride, le rayonnement net est souvent de l'ordre de $500 \mathrm{Wm}^{2}$ et les échanges de chaleur entre le couvert et l'atmosphère se font en régime de convection libre. Ils préconisent l'équation suivante pour calculer le flux $\mathrm{H}$ :

$$
\mathrm{H}=\alpha\left[|\Delta \mathrm{T}|_{z 1}^{\nabla 2}\right]^{3 / 2}
$$

pour $\mathrm{z} 1=0,5 \mathrm{~m}$ et $\mathrm{z} 2=2 \mathrm{~m} \alpha=175$.

Le rapport $H / R n$ pourra être obtenu au $1^{\text {er }}$ ordre par :

$$
\frac{175}{500}\left[\mid \Delta \mathrm{T}_{7.1}^{\not 2}\right]^{3 / 2}
$$

$\mathrm{Ce}$ qui conduit à avoir $\mathrm{H} / \mathrm{Rn}$ voisin de 1 pour $\left.\Delta \mathrm{T}\right|_{0,5 \mathrm{~m}} ^{2 \mathrm{~m}}=2^{\circ} \mathrm{C}$.
En pratique, pour améliorer la précision, la mesure de $\Delta \mathrm{T}$ se fait au moyen d'un dispositif portable simple composé de 12 thermocouples (6 à chaque niveau) placés à 2 hauteurs fixes $(0,5 \mathrm{~m}$ et $2 \mathrm{~m})$.

\section{Mesure de la température radiative}

Deux méthodes ont été retenues pour suivre la température radiative des parcelles sèches et irriguées.

- La première consiste à mesurer à l'échelle horaire, la différence de température entre les parcelles irriguée et non irrigué ( $\mathrm{Tn}$ - Ti) chaque valeur horaire correspond généralement à la moyenne de 4 à 5 mesures. Celles-ci sont réalisées au moyen d'un radiothermomètre différentiel portatif à lecture directe MIKRON. La méthode de mesure consiste à mesurer l'écart de température radiative de chacune des parcelles à une référence identique constituée par un récipient thermostaté rempli d'eau. Ceci permet d'éliminer les erreurs systématiques de la mesure de l'écart de température entre parcelles. De plus, afin de ne prendre en compte que la température des feuilles de tomate et non celle du sol, l'incidence de visée est proche de $30^{\circ}$. Par ailleurs l'azimut de visée est identique, tant par rapport à l'orientation des rangs qui est la même sur les différentes parcelles que par rapport au soleil, afin de comparer des choses comparables.

- La deuxième, plus élaborée, introduit les écarts de température précités dans le calcul des indices de sécheresse. Celui que nous avons retenu, le CWSI (Crop Water Stress Index) a été proposé par IDSo et al. (1981). Les valeurs de CWSI sont déterminées par la relation suivante :

$$
\text { CWSI }=\frac{T n T i}{T \max -\mathrm{Ti}} \simeq 1-\frac{\mathrm{ETR}}{\mathrm{ETP}} .
$$

Avec: Tn : température radiative du couvert non irrigué en ${ }^{\circ} \mathrm{C}$,

Ti : température radiative du couvert irrigué en ${ }^{\circ} \mathrm{C}$,

Tmax : température radiative du même couvert si les plantes ne transpirent pas (couvert complètement desséché) en ${ }^{\circ} \mathrm{C}$.

Nous avons essayé sans succès de mesurer directement Tmax en utilisant des antitranspirants. Ce paramètre a donc été calculé en considérant l'égalité pour un couvert complètement desséché entre le rayonnement net et le flux de chaleur convectif, soit :

$$
H=R n=\rho C p \frac{T \max \cdot T a}{r a}
$$

avec: $\quad \rho$ : masse volumique de l'air $\left(\mathrm{kg} \cdot \mathrm{m}^{3}\right)$,

$\mathrm{Cp}$ : chaleur spécifique de l'air $\left(\mathrm{J} \cdot \mathrm{kg}^{-1} \mathrm{~K}^{\mathrm{l}}\right)$,

Ta: température de l'air mesurée à $2 \mathrm{~m}$ audessus de la végétation $\left({ }^{\circ} \mathrm{C}\right)$,

ra : résistance aérodynamique de l'air mesurée entre la hauteur de végétation et $2 \mathrm{~m}$ audessus de la végétation $\left(\mathrm{s} \cdot \mathrm{m}^{\mathrm{l}}\right)$.

En pratique $\mathrm{Rn}$ et Ta sont mesurables directement, ra est calculée à partir des vitesses de vent à $2 \mathrm{~m}$ et de la hauteur de végétation selon une formule proposée par ITIER \& KATERJI (1983). 
TABLEAU 2

Régimes dirrigation des parcelles étudiées. Time table of water applications.

\begin{tabular}{|c|c|c|c|c|c|c|c|c|c|c|c|c|c|c|c|c|c|c|c|}
\hline Date & $28 / 6$ & 29 & 30 & $1 / 7$ & 2 & 3 & 4 & 5 & 6 & 7 & 8 & 9 & 10 & 11 & 12 & 13 & 14 & 15 & 16 \\
\hline Parcelle $n^{\circ} 1$ & 1 & & & I & $\mathrm{P}$ & & & I & & & I & & I & & I & & & & I \\
\hline Parcelle $n^{\circ} 2$ & $I$ & & & I & $\mathrm{P}$ & & & & & & & & 1 & & I & & & & I \\
\hline Parcelle $n^{\circ} 3$ & I & & & I & $P$ & & & I & & & I & & & & & & & & I \\
\hline
\end{tabular}

$I=$ irrigation.

$\mathrm{P}=$ pluie $7 \mathrm{~mm}$.

\section{Mode d'arrosage et protocole expérimental}

La culture de tomate était effectuée sur une surface de 2 hectares selon les pratiques locales, en rangs d'un mètre, séparés par des raies et par des canaux d'irrigation perpendiculaires à ces raies tous les 50 mètres. Le mode d'arrosage adopté est l'arrosage à la raie : l'eau est pompée d'abord vers les canaux d'irrigation, puis dirigée manuellement vers les raies. L'étude comparative porte sur 3 parcelles qui correspondent à 3 traitements d'irrigation différents (cf. tabl. 2) :

- La parcelle 1 (l ha) a été irriguée pratiquement tous les 2 jours, ainsi elle ne subit pas de déficit hydrique important (comblement de la réserve hydrique dès que la consommation en eau de la culture déterminée par la méthode aérodynamique simplifiée (ITIER, 1981) dépasse 20 p. 100 de la réserve utile dans les premiers $40 \mathrm{~cm}$ du sol.

- La parcelle 2 (0.5 ha) a été laissée en phase de dessèchement pendant 5 jours après une période d'irrigation (établissement d'un point 0 comparatif), puis à nouveau irriguée.

- La parcelle $3(0,2$ ha) a subi le même traitement que la parcelle 2 à 5 jours d'intervalle pour pouvoir répéter l'essai.

Une journée type comporte donc sur chaque parcelle le suivi de 8 paramètres:

- Le potentiel de base qui correspond à la valeur du potentiel hydrique foliaire mesurée à $5 \mathrm{~h} 30$, heure locale (soit $4 \mathrm{~h}$, heure solaire) juste avant le lever du soleil.

- Le potentiel hydrique foliaire, la conductance stomatique, la teneur en eau des feuilles, le rapport $\mathrm{H} / \mathrm{Rn}$, le gradient $|\Delta \mathrm{T}|_{0,5 \mathrm{~m}}^{2 \mathrm{~m}}$, l'écart $\mathrm{Tn}$ Ti et le CWSI à $15 \mathrm{~h}$, heure locale (soit $13 \mathrm{~h} 30$, heure solaire).

De plus, le $15 / 7 / 85$, les 8 paramètres précédents ont été étudiés heure par heure au cours de la journée sur 2 parcelles, irriguée et non irriguée.

\section{RÉSULTATS EXPÉRIMENTAUX}

\section{A. Evolution des critères étudiés au cours de la journcee}

Nous avons suivi sur 2 parcelles, l'une irriguée et l'autre privée d'irrigation depuis 7 jours, la variation horaire de quelques critères au cours de la journée du $15 / 7$.
La figure 1 donne l"evolution des valeurs horaires du potentiel hydrique foliaire et de la conductance stomatique. On constate que ces valeurs subissent au cours de la journée une évolution tout à fait classique: une diminution régulière à partir de la première mesure matinale atteignant vers $14-15 \mathrm{~h}$ (heure locale) les valeurs minimales, puis une reprise par la suite. On note également une différence significative entre les valeurs du potentiel de base et de la conductance stomatique observées sur les parcelles irriguée et non irriguée. En revanche, la différence n'est pas significative en ce qui concerne les valeurs minimales du potentiel hydrique foliaire, sauf peut-être à 13 heures.

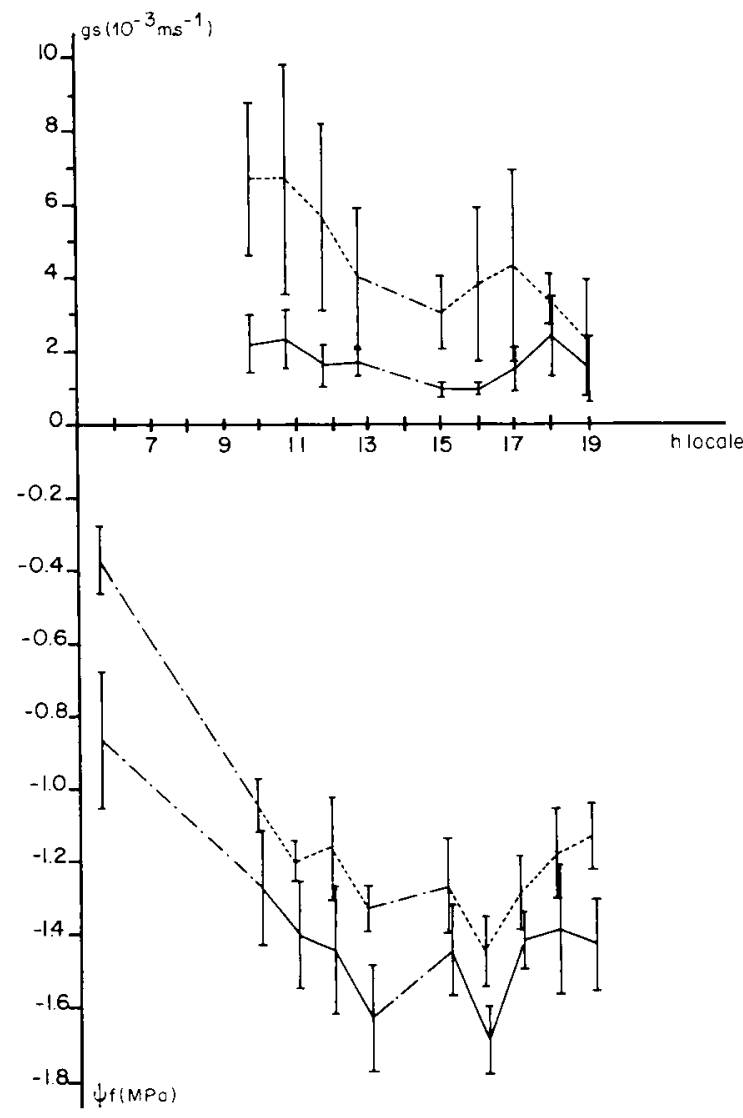

Figure 1

Valeurs horaires, de la conductance stomatique $\left(g_{s}\right)$ et du potentiel hrdrique foliaire $\left(\psi_{F}\right)$ mesurées sur les parcelles irriguées $(-\ldots)$ et non irriguée de tomate (—) au cours de la journée du 15/7/1985. Les traits verticaux correspondent à l'écart-type des mesures.

Mean hourly stomatal conductance $\left(g_{s}\right)$ and mean hourly leaf water potentials $\left(\psi_{F}\right)$ over irrigated ( . . ) and unirrigated (- - ) tomato crop $(15 / 7 / 85)$. The vertical lines correspond to the standard deviation for these measures. 
La figure 2 donne les valeurs horaires de l'écart Tn - Ti et de l'indice CWSI. On note d'abord que les valeurs maximales de ces 2 critères sont atteintes entre 13 et $15 \mathrm{~h}$, ensuite que la fourchette de variation autour des valeurs horaires est importante.

La figure 3 donne les valeurs horaires du flux $\mathrm{H}$ et du gradient $|\Delta \mathrm{T}|_{0,5 \mathrm{~m}}^{2 \mathrm{~m}}$ mesurées sur les parcelles irriguée et non irriguée ainsi que les valeurs horaires du rayonnement net Rn mesuré sur la parcelle irriguée. On observe également ici que les valeurs mesurées entre $13 \mathrm{~h} 30$ et $15 \mathrm{~h}$ correspondent selon le cas aux valeurs maximales (cas de $\mathrm{Rn}$ et $\mathrm{H}$ ) ou aux valeurs minimales (cas de $\left.|\Delta \mathrm{T}|_{0.5 \mathrm{~m}}^{2 \mathrm{~m}}\right)$ observées au cours de la journée.

On note enfin que les résultats présentés figures 1,2 et 3 justifient la démarche adoptée dans le cadre de cette étude et qui consiste à remplacer l'enregistrement continu des critères étudiés par leur prise en compte à $5 \mathrm{~h} 30$ pour le potentiel de base et à $15 \mathrm{~h}$ pour les autres paramètres, c'est-à-dire pendant les périodes où les critères en question atteignent les valeurs extrêmes de la journée.

\section{B. Evolution des critères journaliers étudiés}

A titre d'illustration, nous présentons ici l'évolution des valeurs journalières des critères étudiés sur 2 parcel-

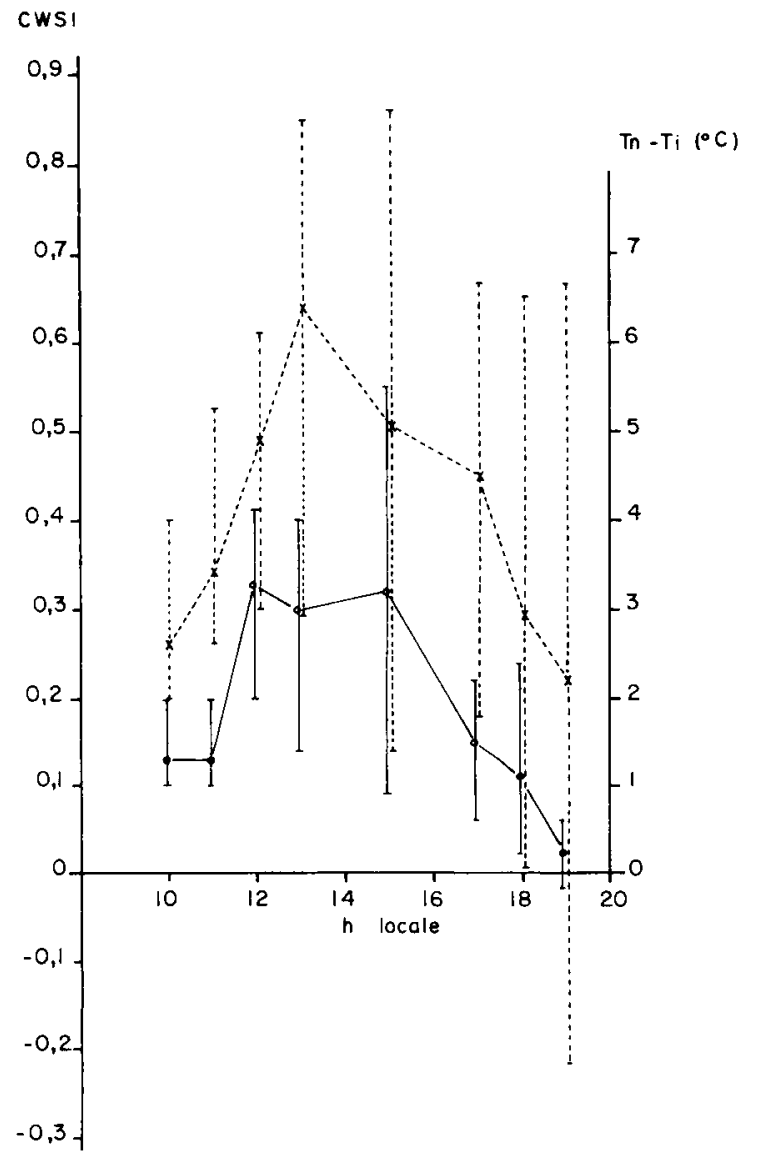

Figure 2

Valeurs horaires, de la différence de temperature (Th-Ti) entre les parcelles irriguée et non irriguée (-), et de l'indice CWSI ( . . .). mesurées au cours de la journée du 15/7/1985.

Les traits verticaux correspondent à l'intervalle maximal de variation.

Hourly variation of radiative temperature difference $(T n-T i)$ between irrigated and unirrigated tomato crop (_- and CWSI (...) (15/7/85).

Vertical lines maximum discrepancy between values.

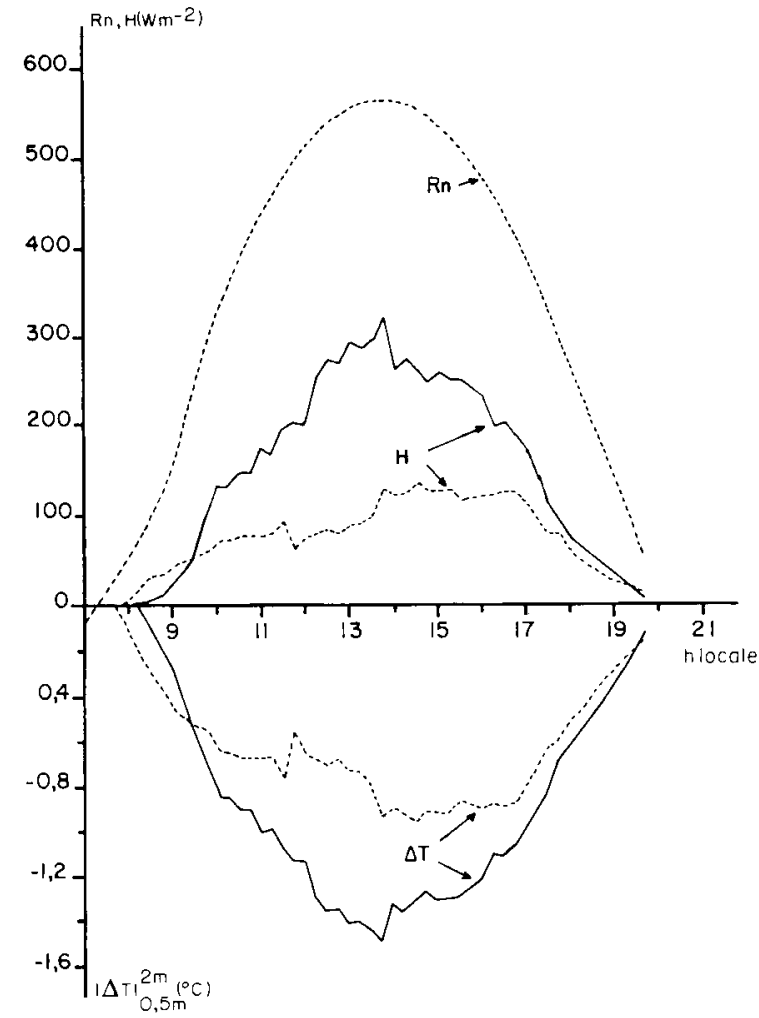

Figure 3

Evolution diurne du rayonnement net $(R n)$ mesuré sur la parcelle irriguée, des flux de chaleur sensible $H$ et des gradients de température dans l'air $|\Delta T|_{0.5 m}^{2 m}$, mestrés sur les parcelles irriguée (-..) at non irriguée (—_ i_ de tomale au cours de la journée du 15/7/1985.

Diurnal change of net radiation Rn over the irrigated plot together with sensible heat flux $H$ and temperature gradient in the air $\Delta T T^{2 m}$, over irrigated ( . . - ) and unirrigated (-) tomato crop (15/7/85)

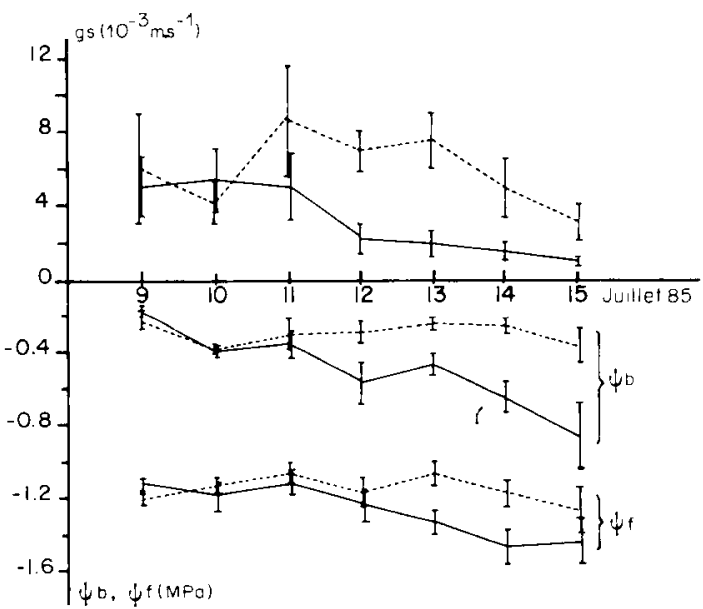

Figure 4

Valours du potenticl de base $\left(\psi_{b}\right)$ mesuries à 5 h 30 , valeurs de la conductance stomatique $\left(g_{s}\right)$ et du potentiel hydrique foliaire $\left(\psi_{F}\right)$ mesurées à 15 h sur les parcelles irriguée ( - . - e ét non irriguée (de tomate au cours de la periode $9-15$ juillet.

Les traits verticaux correspondent à l'écart-type des mesures.

Predann leaf water potential $\left(\psi_{h}\right)$ measured at $05.30 \mathrm{~h}$ and leaf water $\left(\psi_{F}\right)$ potential and stomatal conductance $\left(g_{s}\right)$ measured at $15.00 \mathrm{~h}$ over irrigated (...) and unirrigated (-) tomato crops during Julv 9-15. The vertical lines correspond to the standard deviation for these measures. 
les ( 1 et 3 , cl. tabl. 1). Rappelons que ces 2 parcelles étaient irriguées d'une façon identique jusqu'au 8 juillet, puis que la parcelle 3 a été laissée en dessèchement tandis que la parcelle 1 continuait à être irriguée à intervalles réguliers.

La figure 4 donne l'évolution des valeurs journalières du potentiel de base, de la conductance stomatique et du potentiel hydrique foliaire sur les 2 parcelles en question. On note d'abord, la très bonne concordance entre les valeurs de ces indicateurs observées sur les 2 parcelles avant le dessèchement de la parcelle 3 ; ensuite que la conductance stomatique et le potentiel de base sont des critères beaucoup plus sensibles à l'arrêt de l'irrigation que le potentiel foliaire mesuré à $15 \mathrm{~h}$. Les valeurs concernant la teneur en eau des feuilles n'ont pas été présentées sur la figure 4 dans la mesure où ces valeurs se sont révélées stables et pratiquement du même ordre de grandeur aussi bien sur la parcelle irriguée que sur la parcelle non irriguée pendant toute la période d'observation.

Les valeurs journalières du rapport $\mathrm{H} / \mathrm{Rn}$ et $\mathrm{du}$ gradient $|\Delta \mathrm{T}|_{0.5 \mathrm{~m}}^{2 \mathrm{~m}}$ sont présentées sur la figure 5. Celles de CWSI et de l'écart $\mathrm{Tn}-\mathrm{Ti}$ sont présentées sur la figure 6. On constate que les indicateurs relatifs à la température de surface (CWSI et $\mathrm{Tn}-\mathrm{Ti}$ ) et ceux relatifs aux flux ou gradients aériens $\left(\mathrm{H} / \mathrm{Rn}\right.$ ou $\left.\Delta \mathrm{T}_{(1,5 \mathrm{~m}}^{2 \mathrm{~m}}\right)$ sont relativement aussi sensibles les uns que les autres à un arrêt de l'irrigation. Il est intéressant de noter que les critères simples proposées dans le cadre de cette étude ( $\mathrm{Tn}-\mathrm{Ti})$ et $\left(|\Delta \mathrm{T}|_{0,5 \mathrm{~m}}^{2 \mathrm{~m}}\right)$ sont aussi sensibles que les critères élaborés $(\mathrm{H} / \mathrm{Rn}$ et $\mathrm{CWSI})$.

\section{Evolution des critères étudiés par rapport au potentiel de base}

Le potentiel de base apparaît dans beaucoup d'études expérimentales comme un indicateur très intéressant de l'équilibre sol-plante (MEYER \& GREen, 1980; HaLl \& LARSON, 1982 ; KATERJI \& Hallaire, 1984 ; DWYer \& STEWART, 1985). En plus, contrairement aux autres

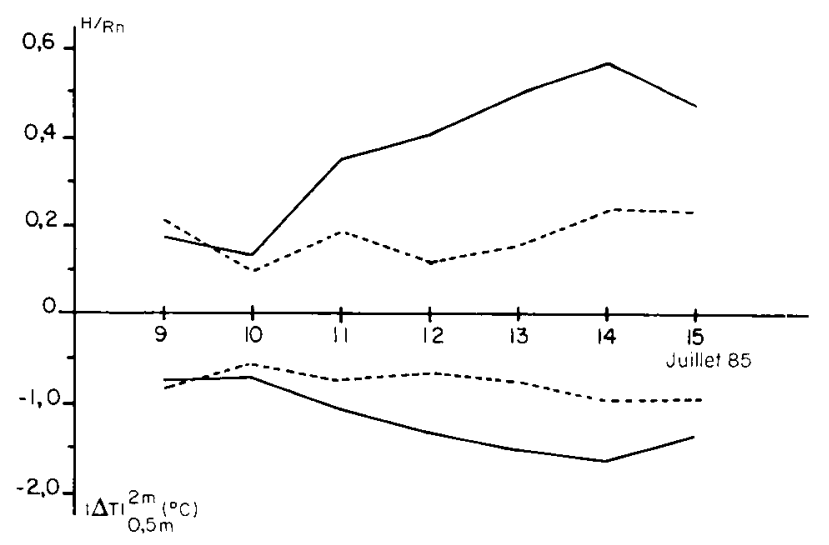

Figure 5

Valeurs du rapport de flux de chaleur sensible au rayonnemem net $(H / R n)$ et du gradien de température dans l'air $\Delta T_{0.5 m}^{2 m}$, mesurces à 15 h sur les parcelles irriguce (- . -) et non irriguée (-) de tomate au cours de la période 9-15 juillet.

Temperature gradient in the air $! \Delta T_{10.5 m}^{2 m}$ and ratio of sensible heat to net radiation $(H / R n)$ measured at $15.00 \mathrm{~h}$ over irrigated (-..) and unirrigated (-) tomato crops during July 9-15.

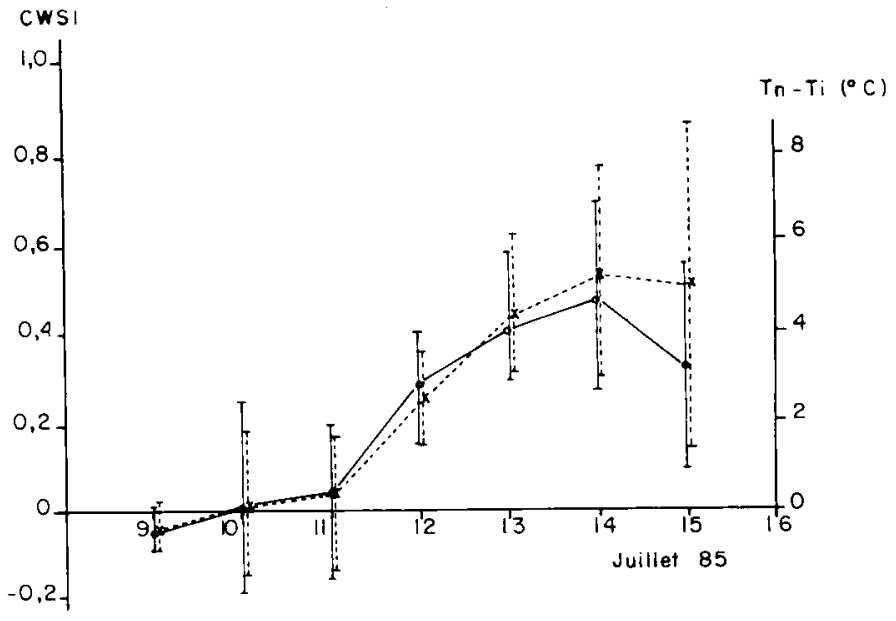

Figure 6

Valcurs de la diffërence de température $\left(T_{n}-T i\right)$ entre les parcelles

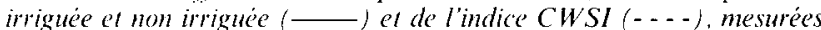
à 15 h au cours de la période 9-15 juillet.

Les traits verticaux correspondent à l'inter valle maximal de variation. CWSI - . -) and radiative temporature difference ( $T n-T i)$ between irrigated and unirigated tomato crops (-) measured at $15.00 \mathrm{~h}$ during $J_{u l}$ y 9-15. The vertical lines comrespond to standard deviation for these measures.

critères de l'état hydrique de la plante, ce paramètre mesuré au lever du soleil est indépendant des conditions micrométéorologiques observées au cours de la journée. Pour cette raison, nous l'avons retenu comme référence pour comparer la sensibilité des paramètres étudiés à un arrêt de l'irrigation.

La figure 7 donne l'évolution des valeurs du potentiel

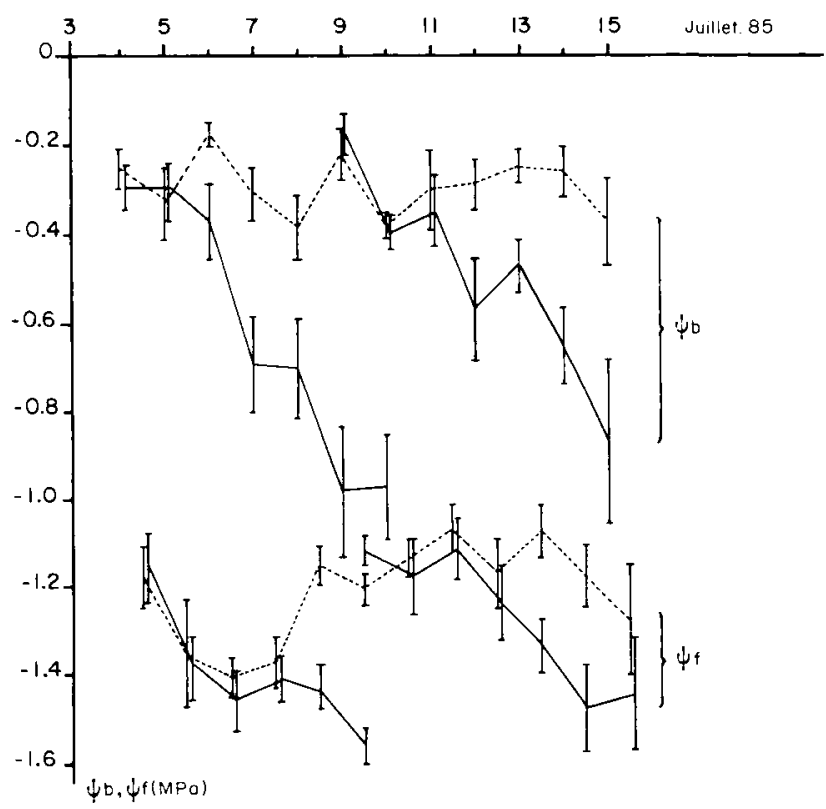

Figure 7

Valeurs du potentiel de hase $\left(\psi_{b}\right)$ et valeurs du potentiel hydrique foliaire $\left(\psi_{r}\right)$ à $15 \mathrm{~h}$ mesurées sur les parcelles irriguée (...) et non irriguée ( $\longrightarrow$ pendant 2 périodes:

$4-9$ juillet (parcelles $1 \& 2$ ).

9-15 juillet (parcelles 1 \& 3 ).

Les traits verticaux correspondent à l'écart-type des mesures.

Predawn leaf water potential $\left(\psi_{h}\right)$ and leaf water $\left(\psi_{F}\right)$ potentials measured at $15.00 \mathrm{~h}$ over irrigated $(\ldots)$ and unirrigated (tomato crops during 2 dry periods :

July 4-9 (plot $\left.n^{\circ} 2\right)$.

July 9-15 (plot $n) 3$ ).

Vertical lines - standard deviations 
de base et du potentiel hydrique foliaire mesuré à $15 \mathrm{~h}$ sur les 3 parcelles. Comme cela a été souligné plus haut (cf. fig. 1 et 4), on note ici que le potentiel de base est un paramètre beaucoup plus sensible que le potentiel foliaire mesuré à $15 \mathrm{~h}$.

La figure 8 donne l'évolution des valeurs mesurées de la conductance stomatique sur les 3 parcelles en fonction du potentiel de base. On constate d'abord que la conductance stomatique diminue avec la diminution du potentiel de base, toutefois les valeurs de conductance deviennent significativement plus faibles à partir des potentiels de base de l'ordre de $-0,4 \mathrm{MPa}$. On note enfin que la dispersion est faible lorsque le potentiel de base diminue, ce qui traduit la dominance de la contrainte hydrique sur le comportement des stomates.

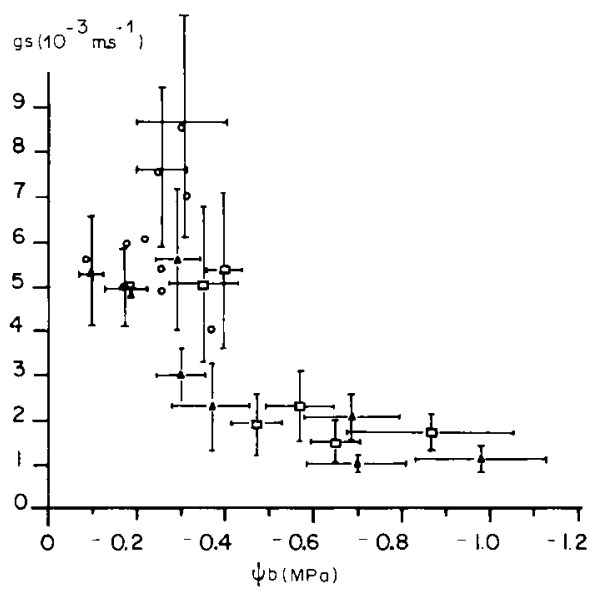

Figure 8

Valeurs de la conductance stomatique (gs) mesurées à 15 h en fonction du potentiel de base ( $\psi b)$ :

sur la parcelle irriguée (parcelle $n^{\circ} 1$ ).

$\triangle \square$ respectivement sur les parcelles non irriguées, $n^{o s} 2 \& 3$

Les traits verticaux et horizontaux correspondent à l'écart-type des mesures.

Stomatal conductance ( $g$ s) measured at $15.00 \mathrm{~h}$ as a function of predawn leaf water potential $\left(\psi_{h}\right)$

irrigated tomato crops (plot $n^{\circ} 1$ ),

$\triangle \square$ unirrigated tomato crops (plots $n^{\circ} 2$ et 3 ).

Vertical and horizontal lines - standard deviations.

L'évolution des 4 critères: CWSI, Tn $-\mathrm{Ti}, \mathrm{H} / \mathrm{Rn}$, $|\Delta \mathrm{T}|_{0.5 \mathrm{~m}}^{2 \mathrm{~m}}$ a été étudiée sur tomate pendant 2 répétitions (période du 4 au 9 et du 9 au 15 juillet). Ces résultats conduisent à plusieurs observations (cf. fig. 9 et 10 ) :

- La relation entre les 4 critères en question et le potentiel de base est relativement bonne en général pendant la période du 9 au 15 juillet, notamment pour le CWSI et $\mathrm{Tn}-\mathrm{Ti}$; en revanche, elle est nettement moins bonne pendant la période du 4 au 9 juillet.

- Pour les 2 périodes étudiées, on note que les critères simples proposés ici $\left(|\Delta \mathrm{T}|_{0,5 \mathrm{~m}}^{2 \mathrm{~m}}\right.$ et $\mathrm{Tn}$ Ti) sont aussi sensibles à un arrêt de l'irrigation que les critères élaborés (H/Rn et CWSI).

- Il est difficile, compte tenu des résultats obtenus, de définir des valeurs critiques pour le déclenchement de l'irrigation pour chacun des paramètres étudiés.
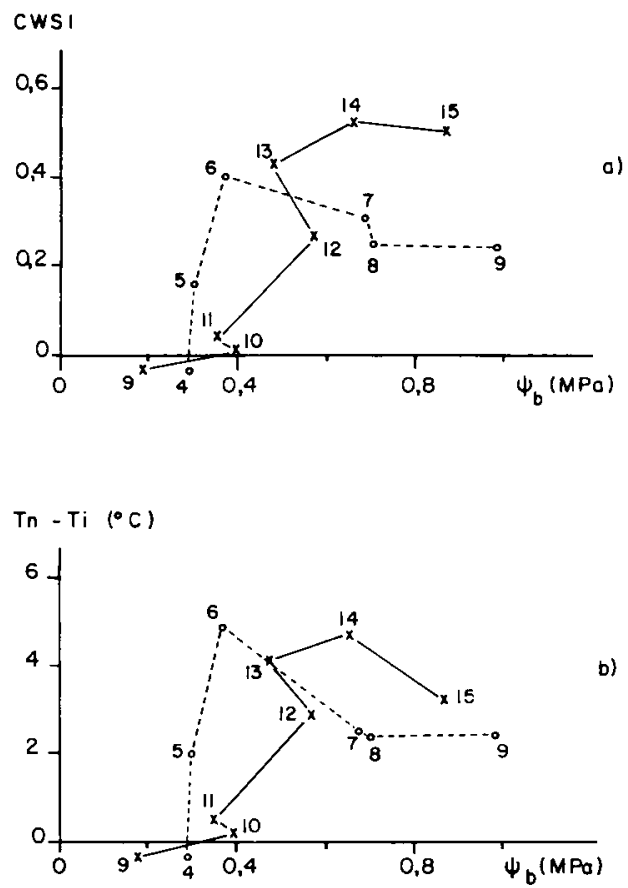

Figure 9

Valeurs mesurées à 15 h de l'indice CWSI (fig. 9 a) et de la différence de tempéraiure ( $T n-T i)$ entre les parcelles non irriguée et irriguée de tomate (fig. $9 \mathrm{~b}$ ) en fonction du potentiel de base $\left(\psi_{b}\right)$ :

- 4-9 juillet (parcelles I \& 2).

- $9-15$ juillet (parcelles 1 \& 3 ).

CWSI (fig. 9a) and radiative temperalure differences $(T n-T i)$ between irrigated and unirrigated tomato crop (fig. 9 b) measured at $15.00 \mathrm{~h}$ as functions of predawn leaf water potential $\left(\psi_{h}\right)$ during 2 dry periods.

July 4-9 (plot $n^{\text {rs }} 1$ \& 2$)$

- July 9-15 (plot nos 1 \& 3 ).

\section{DISCUSSION ET CONCLUSION}

L'ensemble des résultats obtenus dans le cadre de cette étude fournit des éléments de réponse aux objectifs posés au départ.

L'un des objectifs de cette étude était de justifier l'utilisation des critères simples. Nous avons démontré d'abord que le suivi des critères étudiés pendant une partie de la journée au moment où ces critères atteignent les valeurs maximales ou minimales journalières est suffisant pour apprécier le comportement hydrique des cultures; ensuite, lorsque 2 méthodes, l'une élaborée et l'autre simple, sont utilisées pour appréhender le même critère (ex. le rapport $\mathrm{H} / \mathrm{Rn}$ ), l'utilisation de la méthode simple ne semble pas diminuer la sensibilité du paramètre en question.

L'objectif principal de cette étude reste cependant la comparaison entre l'aptitude des critères étudiés à détecter l'état hydrique de la plante suite à un arrêt de l'irrigation; les résultats obtenus sur une culture de tomate fournissent dans ce domaine des indications intéressantes.

Nous avons noté en effet, et contrairement aux conclusions de Kagmar et al. (1980) que, chez la tomate, la conductance stomatique et le potentiel de base sont des critères très sensibles à un arrêt de l'irrigation. Le potentiel hydrique foliaire mesuré à midi semble être au contraire pratiquement insensible. 


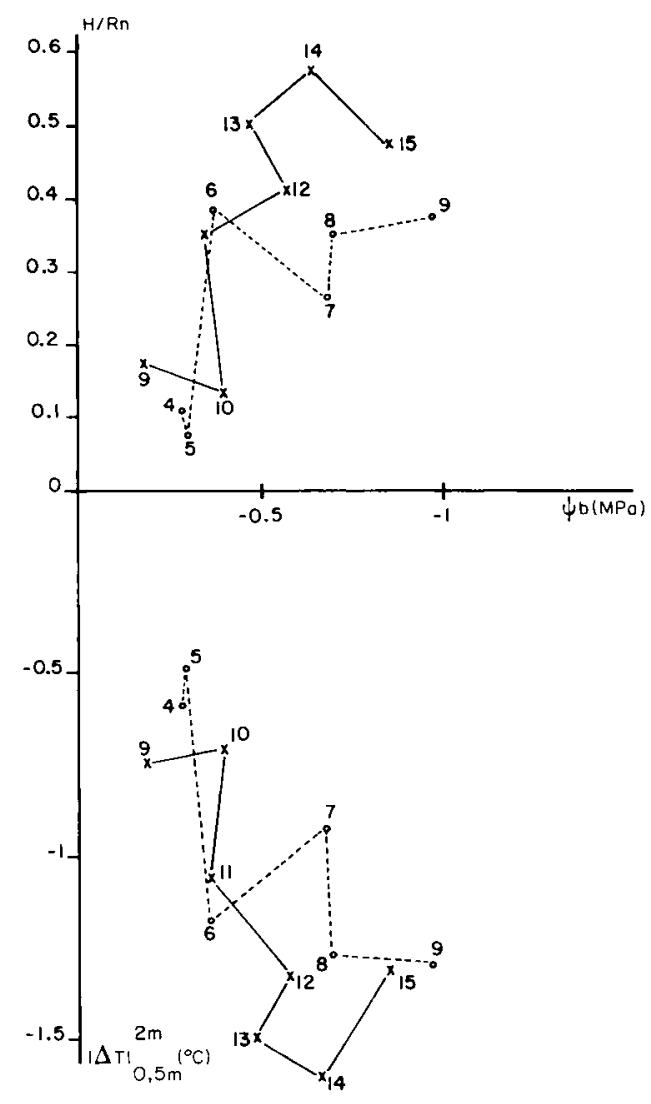

Figure 10

Valeurs mesuries à $15 \mathrm{~h}$ du rapport du flux de chaleur sensible au rayonnement net $(\mathrm{H} / \mathrm{Rn})$ et du gradient de température dans l'air $|\Delta T|_{0.5 m}^{2 n t}$ en fonction du potenticl de base $\left(\psi_{b}\right)$.

-. 4-9 juillet (parcelles 1 \& 2 ).

-9-15 juillet (parcelles 1 \& 3 ).

Ratio of sensible heat to net radiation ( $H / R n)$ and air temperature gradient,$\left.\Delta T\right|_{10,5 m} ^{2 m}$ measured at $15.00 \mathrm{~h}$ as functions of predawn leaf water potential $\left(\psi_{h}\right)$ during 2 dry periods:

-... July 4-9 (plot $\left.n^{2.4} i \& 2\right)$.

Jlily 9-15 (plot nt.. 1 \& 3)

Comme nous l'avons souligné dans l'introduction, certains auteurs arrivent à des conclusions semblables, d'autres, au contraire, considèrent ce critère comme un indicateur particulièrement intéressant. Ces résultats contradictoires peuvent être interprétés à notre avis comme étant une conséquence de la stratégie que l'espèce végétale adopte en conditions de sécheresse. En effet, si l'espèce en question observe un comportement isohydrique (cf. PARCEvaux, 1963), c'est-à-dire qu'elle maintient un potentiel hydrique relativement élevé clans les tissus végétaux pendant la période chaude de la journée, comme la tomate, le potentiel hydrique foliaire ou la teneur en eau ne seraient pas des critères intéressants. Dans le cas d'une espèce ayant un comportement anisohydrique (comportement inverse au cas précédent) le potentiel hydrique foliaire pourrait être au contraire un critère intéressant.

Les résultats concernant les critères relatifs à la température ou au flux de surface montrent que la sensibilité de ces critères peut changer d'une répétition à l'autre.

Plusieurs hypothèses peuvent être évoquées pour expliquer cette différence; on note d'abord que certains critères (ex. gradient $|\Delta \mathrm{T}|_{0.5 \mathrm{~m}}^{23 \mathrm{~m}}$ ) sont sensibles aux phénomènes d'advection; il est donc difficile d'appréhender ces critères sur des parcelles de petites dimensions (inférieures à $1 \mathrm{ha}$ ) en région semi-aride. Une autre source d'erreur est due probablement à la détermination de l'indice de sécheresse CWSI à partir des valeurs calculées de Tmax. Comme nous l'avons déjà souligné plusieurs tentatives pour mesurer directement Tmax sont restées infructueuses.

Les observations réalisées sur une culture de tomate nous conduisent à considérer la conductance stomatique et le potentiel de base comme des critères particulièrement performants. Le potentiel de base ayant l'avantage d'être sûr, d'être réalisé au moyen d'un matériel peu coûteux ( $<20 \mathrm{KF}$ ), de nécessiter moins de précautions et d'être déterminé à une heure de la journée où le comportement isohydrique ou anisohydrique de la culture ne se manifeste pas encore. Les mesures liées à la température de surface ou au flux de chaleur ne sont intéressantes que si certaines précautions relatives à la dimension de la parcelle étudiée sont prises en considération.

Les observations concernant le potentiel de base, bien que confirmées sur d'autres espèces végétales (DWYER \& Stewart, 1985; SCHOCH et al., 1987) doivent être nuancées dans certaines situations. GARNIER \& BERGER (1987) ont noté, dans le cas des espèces ayant des réserves hydriques importantes (arbres fruitiers), cultivées sur des sols argileux, que le potentiel de base est nettement moins sensible que les autres indicateurs comme la contraction journalière du diamètre du tronc. Il sera donc raisonnable de réaliser des expérimentations comparables à celles présentées ici sur plusieurs espèces cultivées et sur plusieurs types de sol avant de généraliser l'utilisation du potentiel de base.

Reçu le 16 septembre 1987 Accepté le 17 mars 1988.

\section{RÉFÉRENCES BIBLIOGRAPHIQUES}

Bordovsky D. G., Jordan W. R., Hiler E. A., Howell T. A., 1974. Choicc of irrigation timing indicator for narrow row cotton. Agron. J., $66,88-91$

Bouchet R. J., Robelin M., 1969. Evapotranspiration potentielle et réelle. Domaine d'utilisation-portée pratique. Bull. Techn. Inf., 238 , 215-223.

Clark R. N., Hiler E. A., 1973. Plant measurements as indicators of crop water deficits. Crop Sci., 13, 466-469.

Dwyer L. M., Stewart D. W., 1985. Indicators of water stress in corn (Zea ma!s L.). Can. J. Plant Sci., 64, 537-546.
Ferreira I., 1987. Evapotranspiraçao real : estudo realizado na cultura do tomate em regiao de clima mediterrânico. Thèse Doctorat, Universidade Tecnica de Lisboa, Instituto superior de agronomia. $168 \mathrm{p}$.

Garnier E., 1985. Dessèchement du sol et indicateurs physiologiques de l'état hydrique chez le pêcher: application au déclenchement de l'irrigation. Thèse Docteur-Ingénieur, INSA Montpellier, $82 \mathrm{p}+$ annexes.

Garnier E., Berger A., 1987. The influence of drought on stomatal conductance and water potential of peach trees growing in the field. Sci Hort., 32, 249-263.

Hall R. G., Larson K. L., 1982. Water stress of alfalfa during stress and recovery. Can. J. Plant Sci., 62, 639-647. 
Idso S. B., Reginato R. J., Jackson R. D., Pinter P. J., 1981. Foliage and air temperatures: evidence for a dynamic equivalent point. Agric. Meteorol., 24, 223-226.

Itier B., 1981. Une méthode simple pour la mesure de l'évapotranspiration réelle à l'échelle de la parcelle. Agronomie. 1, 869-876.

Itier B., Katerji N., 1983. Une expression simplifiée de la résistance aérodynamique d'un couvert végétal. Acta Oecol. Oecol. general., 3, 215-227.

Itier B., Riou Ch., 1985. Une possibilité de suivi de l'état hydrique des cultures en zone semi-aride à partir de mesures micrométéorologiques simples. In : Les besoins en eau des cultures, Perrier A. \& Riou Ch. ed., Coll. C.I.I.D. Paris, $831-840$

Jackson R. D., 1982. Canopy temperature and crop water stress. In : Advances in irrigation. Hillel D. ed., Vol. 1, Academic Press New York, 43-85.

Kamgar A. A., Henderson D. W., Pruitt W. O., 1980. Evaluating leaf water potential, stomatal resistance, and canopy surface temperature of tomatoes as indices for irrigation timing. Acta Hort. 100, 181-192.

Katerji N., Hallaire M., 1984. Les grandeurs de référence utilisables dans l'étude de l'alimentation en eau des cultures. Agronomie, 4, 999-1008.

Kozlowski T. T., 1968. Diurnal changes in diameters of fruit and tree stems of Montmorency cherry. J. Hort. Sci., 43, 1-15.

Ludlow M. M., 1976. Ecophysiology of C4 grasses. In : Water and plant life Lange et al., ed., Springer, Berlin, 364-386.
Meyer W. S., Green G. C., 1980. Water use by wheat and plant indicators of available soil water. Agron. J., 72, 253-257.

Namken L. N., 1965. Relative turgidity technique for scheduling cotton (Gossypium hirsutum) irrigation. Agron. J., 57, 38-41.

Namken L. N., Bartholic J. F., Runkles J. R., ( 1971 ). Water stress and stem radial contraction of cotton plants (Gossypium hirsutum L.) under field conditions. Agron. J., 63, 623-627.

O'Toole J. C., Moya T. B., 1978. Genotypic variation in maintenance of leaf water potential in rice. Crop Sci., 18, 873-876.

O'Toole J. C., Turner N. C., Namuco O. P., Din Gkuhn M., Gomez K. A., 1984. Comparison of some crop water stress measurement methods. Crop Sci., 24, 1121-1128.

Parcevaux S. de, 1963. Transpiration végétale et production de matière sèche. Ann. Agron., 14, 655-742.

Ritchie J. T., Jordan W. R., 1972. Dryland evaporative flux in a subhumid climate: IV - Relation to plant water status. Agron. J., 2 173-176.

Schoch P. G., Katerji N., Rimgoto P., Tchamitchian M., Malet P., 1987. Influence du niveau d'alimentation hydrique sur les variations du diamètre des tiges, du potentiel hydrique, de la résistance stomatique, de la transpiration et de la photosynthèse de l'aubergine (Solanum melongena L.). Agric. Forest Meteorol., 40, 89-104.

Scholander P. F., Hammel H. T., Bradsteet E. D., Hemmingsen E. A., 1965. Sap pressure in vascular plants. Science, 148, 339-346.

Stegman E. C., Schiele L. H., Bauer A., 1976. Plant water stress criteria for irrigation scheduling. Trans. A.S.A.E., 19, 850-855. 